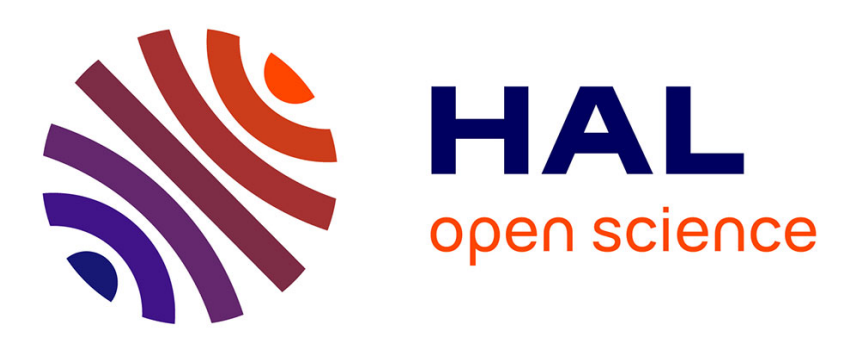

\title{
State and unknown input estimation for nonlinear systems described by Takagi-Sugeno models with unmeasurable premise variables
}

\author{
Dalil Ichalal, Benoît Marx, José Ragot, Didier Maquin
}

\section{To cite this version:}

Dalil Ichalal, Benoît Marx, José Ragot, Didier Maquin. State and unknown input estimation for nonlinear systems described by Takagi-Sugeno models with unmeasurable premise variables. 17th Mediterranean Conference on Control and Automation, MED'09, Jun 2009, Thessaloniki, Greece. pp.353-358, 10.1109/MED.2009.5164542 . hal-00376460

\author{
HAL Id: hal-00376460 \\ https://hal.science/hal-00376460
}

Submitted on 31 Mar 2014

HAL is a multi-disciplinary open access archive for the deposit and dissemination of scientific research documents, whether they are published or not. The documents may come from teaching and research institutions in France or abroad, or from public or private research centers.
L'archive ouverte pluridisciplinaire HAL, est destinée au dépôt et à la diffusion de documents scientifiques de niveau recherche, publiés ou non, émanant des établissements d'enseignement et de recherche français ou étrangers, des laboratoires publics ou privés. 


\title{
State and unknown input estimation for nonlinear systems described by Takagi-Sugeno models with unmeasurable premise variables
}

\author{
Dalil Ichalal, Benoît Marx, José Ragot, Didier Maquin
}

\begin{abstract}
This paper presents a new method to synthesize observers for continuous time nonlinear systems described by Takagi-Sugeno (TS) model with unmeasurable premise variables. First, convergence conditions are established in order to guarantee the convergence of the state estimation error. These conditions are given in Linear Matrix Inequality (LMI) formulation. Secondly, a classical Proportional Integral Observer (PIO) is extended to the considered nonlinear systems in order to estimate the state and the unknown inputs (UI).
\end{abstract}

\section{INTRODUCTION}

Recently, monitoring and diagnosis of nonlinear systems took an important consideration. Indeed, the unceasing demand in terms of reliability and performance of systems has led to the use of nonlinear models to represent the systems. Therefore obtained models are very complex and the task of model-based fault diagnosis becomes more difficult to achieve.

In recent years, the proposed Takagi-Sugeno structure introduced in [1] provides a better representation of nonlinear systems in terms of mathematical complexity. Thus, highly nonlinear behaviors can be represented by simple models. The Takagi-Sugeno model structure, sometimes known as multiple model structure, is based on the decomposition of the operating range in several zones (operating points) and the behavior of the system in each zone is represented by a local linear model. Thanks to an appropriate choice of the weighting functions, the blending of the local models can efficiently represents the overall behavior of the system. The contribution of each local model is quantified by the weighting functions. These nonlinear functions verify the property of convex sum. This important property allows the extension of some analysis and design tools developed in the linear system framework to nonlinear systems, which is the main interest of the Takagi-Sugeno structure for the study of nonlinear systems.

Concerning the state estimation of nonlinear systems represented by Takagi-Sugeno models, we can cite [2], [3], [4], where the authors extended the Luenberger observer and the unknown input observer (UIO) to nonlinear systems. These two observers are used in [4] and [5] to develop an observer bank based method to detect and isolate actuator and sensor faults. Another model-based approach to fault

This research was supported by the TASSILI No. 07 program under MDU grant 714 .

All the authors are with the Centre de Recherche en Automatique de Nancy (CRAN) UMR 7039, Nancy-Université, 2 , avenue de la forêt de Haye 54516 Vandoeuvre-les-Nancy \{dalil.ichalal, benoit.marx, jose.ragot,

didier.maquin\}eensem. inpl-nancy. fr detection for Takagi-Sugeno models is proposed in [6], where the authors use the techniques of sensitivity constraints to detect and isolate the faults. In the works cited above, the authors assume that the weighting functions depend on measurable premise variables (like the input or the output of the system). The case where the weighting functions depend on unmeasurable premise variables (like the state of the system) is studied in [7], [8], [9]. The authors propose an observer which is an extension of the Thau-Luenberger observer [10] and they give a methodology for synthesizing the gains of the observer using an LMI approach.

Section II gives some notations used in the paper, presents the Takagi-Sugeno model structure and finally states the studied problem. Sections III and IV present the main results concerning the design of observer to estimate the state and the unknown inputs of nonlinear systems described by Takagi-Sugeno models. Before concluding, simulation results are given in section $\mathrm{V}$.

\section{NOTATION AND PROBLEM STATEMENT}

In this paper the following notations are used

$$
\sum_{i=1}^{r} \sum_{j=1}^{r} \sum_{k=1}^{r} \mu_{i} \mu_{j} \mu_{k} \Leftrightarrow \sum_{i, j, k=1}^{r} \mu_{i} \mu_{j} \mu_{k}
$$

$I_{n}$ is an $n \times n$ identity matrix.

\section{A. Takagi-Sugeno model}

Let us consider the TS model representation of a nonlinear system given by

$$
\left\{\begin{array}{l}
\dot{x}(t)=\sum_{i=1}^{r} \mu_{i}(\xi(t))\left(A_{i} x(t)+B_{i} u(t)\right) \\
y(t)=\sum_{i=1}^{r} \mu_{i}(\xi(t))\left(C_{i} x(t)+D_{i} u(t)\right)
\end{array}\right.
$$

where $x(t) \in \mathbb{R}^{n}$ is the state vector, $u(t) \in \mathbb{R}^{m}$ is the control input and $y(t) \in \mathbb{R}^{p}$ is the measurement output. $A_{i}, B_{i}, C_{i}$ and $D_{i}$ are real known matrices with appropriate dimensions. The weighting functions $\mu_{i}$ are nonlinear in $\xi(t)$ and satisfy the convex sum property:

$$
\left\{\begin{array}{l}
\sum_{i=1}^{r} \mu_{i}(\xi(t))=1 \quad \forall t \\
0 \leq \mu_{i}(\xi(t)) \leq 1, i \in\{1, \ldots, r\} \quad \forall t
\end{array}\right.
$$

The premise variable $\xi(t)$ can depend on measurable signals, for example the input $u(t)$ or the output $y(t)$ of the system (this situation is largely studied in the literature), or on unmeasurable signals like the state $x(t)$. 


\section{B. Problem statement}

Takagi-Sugeno model has proved its effectiveness in the study of nonlinear systems. In the case of bounded nonlinearities, TS structure not only provides a mathematically equivalent form, but also highlights each of the linear submodels [1]. In the field of stability analysis and stabilization, many works, such as state feedback control [2], [11], [12], [13], [3] have been developed and applied in a lot of practical situations. The problem of state estimation has also been studied in order to design state feedback control laws and to design a residual generator in order to detect and isolate faults in the system and to reconfigure the control laws in the presence of faults [4], [7].

In the field of state estimation and diagnosis of nonlinear systems using multiple model approach, the most of the published works considered TS models with measurable premise variables [4], [14]. It is clear that the choice of measurable premise variables offers a good simplicity to generalize the methods already developed for linear systems. But in the case where the premise variables are not measurable, the problem becomes very hard. However, this formalism is very important both in the exact representation of the nonlinear behavior and in diagnosis method based on observer banks to detect and isolate actuator and sensor faults. Indeed in this case, the use of measurable premise variables requires to develop two different multiple models, the first using the input $u(t)$ in the premise variable to detect and isolate sensor faults, and the second using the output of the system for actuator faults. Considering unmeasurable premise variables allows to develop only one multiple model of the system behavior to detect and isolate both actuator and sensor faults using observer banks. In the literature, few works are devoted to the case of unmeasurable premise variables, nevertheless, we can cite [7], [15], [8], where the authors proposed the fuzzy Thau-Luenberger observer which is an extension of the classical Luenberger observer. The main contribution of this paper is to propose a method to estimate not only the state variables, but also the unknown input affecting the system. Our approach allows to reduce the conservatism, linked to Lipschitz conditions, of the existing works [7] and relax the conditions under which the method is applicable. The proposed method is given for more general T-S systems because it includes the case where the output of the system is nonlinear with regard to the state of the system.

\section{State estimation In the UI FReE CASE}

Consider the system (1) with weighting functions depending on the state of the system:

$$
\left\{\begin{array}{l}
\dot{x}(t)=\sum_{i=1}^{r} \mu_{i}(x(t))\left(A_{i} x(t)+B_{i} u(t)\right) \\
y(t)=\sum_{i=1}^{r} \mu_{i}(x(t))\left(C_{i} x(t)+D_{i} u(t)\right)
\end{array}\right.
$$

For the sake of simplicity, $(t)$ will be omitted in the sequel.

Let us denote the estimated state by $\hat{x}$. By adding and subtracting the term $\sum_{i=1}^{r}\left(\mu_{i}(x)-\mu_{i}(\hat{x})\right)\left(A_{i} x+B_{i} u\right)$, we obtain the following equivalent system:

$$
\begin{aligned}
\dot{x} & =\sum_{i=1}^{r} \mu_{i}(\hat{x})\left(A_{i} x+B_{i} u\right) \\
& +\sum_{i=1}^{r}\left(\mu_{i}(x)-\mu_{i}(\hat{x})\right)\left(A_{i} x+B_{i} u\right)
\end{aligned}
$$

As explained in the previous section, thanks to (2), we have:

$$
\sum_{i=1}^{r}\left(\mu_{i}(x)-\mu_{i}(\hat{x})\right) X_{i}=\sum_{i, j=1}^{r} \mu_{i}(x) \mu_{j}(\hat{x})\left(X_{i}-X_{j}\right)
$$

where $X_{i} \in\left\{A_{i}, B_{i}, C_{i}, D_{i}\right\}$. Let us define the following notations:

$$
\Delta X_{i j}=X_{i}-X_{j}
$$

Then the system (4) can be transformed into the following system:

$$
\dot{x}=\sum_{i, j=1}^{r} \mu_{i}(x) \mu_{j}(\hat{x})\left(\left(A_{j}+\Delta A_{i j}\right) x+\left(B_{j}+\Delta B_{i j}\right) u\right)
$$

The output equation can similarly be written in the following form:

$$
y=\sum_{i, k=1}^{r} \mu_{i}(x) \mu_{k}(\hat{x})\left(\left(C_{k}+\Delta C_{i k}\right) x+\left(D_{k}+\Delta D_{i k}\right) u\right)
$$

The system (7)-(8) is written like an uncertain system but the considered "uncertain terms" $\Delta X_{i j}$ are completely known and are constant matrices.

The proposed observer is given by the following equations:

$$
\left\{\begin{array}{l}
\dot{\hat{x}}=\sum_{j=1}^{r} \mu_{j}(\hat{x})\left(A_{j} \hat{x}+B_{j} u+G_{j}(y-\hat{y})\right) \\
\hat{y}=\sum_{k=1}^{r} \mu_{k}(\hat{x})\left(C_{k} \hat{x}+D_{k} u\right)
\end{array}\right.
$$

Taking (2) into account, the equations (8) can be multiplied by $\sum_{i=1}^{r} \mu_{i}(x)$ to obtain:

$$
\begin{gathered}
\dot{\hat{x}}=\sum_{i, j=1}^{r} \mu_{i}(x) \mu_{j}(\hat{x})\left(A_{j} \hat{x}+B_{j} u+G_{j}(y-\hat{y})\right) \\
\hat{y}=\sum_{i, k=1}^{r} \mu_{i}(x) \mu_{k}(\hat{x})\left(C_{k} \hat{x}+D_{k} u\right)
\end{gathered}
$$

The weighting functions $\mu_{i}(x)$ formally appear in (10-11) although they are not available since $x$ is not known. But it should be noticed that since no term is indexed on $i$ in (10-11) the computations of $\hat{x}$ and $\hat{y}$ are still feasible.

The state estimation error is given as follows:

$$
e=x-\hat{x}
$$

Using (7), (8), (10) and (11), the dynamics of the state estimation error is:

$$
\dot{e}=\sum_{i, j, k=1}^{r} \mu_{i}(x) \mu_{j}(\hat{x}) \mu_{k}(x)\left(\Phi_{j k} e+\Gamma_{i j k} x+S_{i j k} u\right)
$$

where:

$$
\begin{aligned}
& \Phi_{j k}=A_{j}-G_{j} C_{k} \\
& \Gamma_{i j k}=\Delta A_{i j}-G_{j} \Delta C_{i k} \\
& S_{i j k}=\Delta B_{i j}-G_{j} \Delta D_{i k}
\end{aligned}
$$




$$
i, j, k \in\{1, \ldots, r\}
$$

Let us define the augmented state $\tilde{x}=\left[\begin{array}{ll}e^{T} & x^{T}\end{array}\right]^{T}$ which dynamics is described by the augmented system:

$$
\begin{aligned}
\dot{\tilde{x}} & =\sum_{i, j, k=1}^{r} \mu_{i}(x) \mu_{j}(\hat{x}) \mu_{k}(x)\left(\mathscr{M}_{i j k} \tilde{x}+\mathscr{B}_{i j k} u\right) \\
z & =H \tilde{x}
\end{aligned}
$$

where:

$$
\mathscr{M}_{i j k}=\left[\begin{array}{cc}
\Phi_{j k} & \Gamma_{i j k} \\
0 & A_{i}
\end{array}\right], \mathscr{B}_{i j k}=\left[\begin{array}{c}
\Sigma_{i j k} \\
B_{i}
\end{array}\right], H=\left[\begin{array}{ll}
I_{n} & 0
\end{array}\right]
$$

The goal then is to determine $G_{j}$ to guarantee the stability of (14) while attenuating the effect of the input $u(t)$ on $z(t)$.

Theorem 1: The system (14) is stable and the $\mathscr{L}_{2}$-gain of the transfer from $u(t)$ to $z(t)$ is bounded, if there exists symmetric matrices $P_{1}$ and $P_{2}$, matrices $K_{i}$ and a positive scalar $\bar{\gamma}$, such that the following conditions hold:

$$
\left[\begin{array}{ccc}
X_{1 j k} & \Theta_{i j k} & \Psi_{i j k} \\
\Theta_{i j k}^{T} & X_{2 i} & P_{2} B_{i} \\
\Psi_{i j k}^{T} & B_{i}^{T} P_{2} & -\bar{\gamma} I
\end{array}\right]<0, \quad \forall(i, j, k) \in\{1, \ldots, r\}^{3}
$$

where:

$$
\begin{aligned}
X_{1 j k} & =A_{j}^{T} P_{1}+P_{1} A_{j}-K_{j} C_{k}-C_{k}^{T} K_{j}^{T}+I \\
X_{2 i} & =A_{i}^{T} P_{2}+P_{2} A_{i} \\
\Theta_{i j k} & =P_{1} \Delta A_{i j}-K_{j} \Delta C_{i k} \\
\Psi_{i j k} & =P_{1} \Delta B_{i j}-K_{j} \Delta D_{i k}
\end{aligned}
$$

The gains of the observer are derived from:

$$
G_{j}=P_{1}^{-1} K_{j}
$$

and the attenuation level is :

$$
\gamma=\sqrt{\bar{\gamma}}
$$

Proof: Considering the following quadratic Lyapunov function:

$$
V(\tilde{x})=\tilde{x}^{T} P \tilde{x}, \quad P=P^{T}>0
$$

its derivative with regard to time is given by:

$$
\dot{V}(\tilde{x})=\dot{\tilde{x}}^{T} P \tilde{x}+\tilde{x}^{T} P \dot{\tilde{x}}
$$

By substituting $\dot{\tilde{x}}(14)$ in (25), we obtain:

$$
\begin{aligned}
\dot{V}(\tilde{x}) & =\sum_{i, j, k=1}^{r} \mu_{i}(x) \mu_{j}(\hat{x}) \mu_{k}(x)\left(\tilde { x } ^ { T } \left(\mathscr{M}_{i j k}^{T} P\right.\right. \\
& \left.\left.+P \mathscr{M}_{i j k}\right) \tilde{x}+\tilde{x}^{T} P \mathscr{B}_{i j k} u+u^{T} \mathscr{B}_{i j k}^{T} P \tilde{x}\right)
\end{aligned}
$$

The goal is to attenuate the effect of the input $u(t)$ on $z(t)$. So, in order to guarantee the stability of (13) and the boundedness of the transfer from $u(t)$ to $z(t)$ :

$$
\frac{\|z(t)\|_{2}}{\|u(t)\|_{2}}<\gamma, \quad\|u(t)\|_{2} \neq 0, \quad \gamma>0
$$

we consider the following criterion:

$$
\dot{V}(\tilde{x})+z^{T} z-\gamma^{2} u^{T} u<0
$$

Substituting (26) and (15) in (28), we obtain:

$$
\begin{array}{r}
\sum_{i, j, k=1}^{r} \mu_{i}(x) \mu_{j}(\hat{x}) \mu_{k}(x)\left(\tilde{x}^{T}\left(\mathscr{M}_{i j k}^{T} P+P \mathscr{M}_{i j k}\right) \tilde{x}\right. \\
\left.+\tilde{x}^{T} P \mathscr{B}_{i j k} u+u^{T} \mathscr{B}_{i j k}^{T} P \tilde{x}\right)+\tilde{x}^{T} H^{T} H \tilde{x}-\gamma^{2} u^{T} u<0
\end{array}
$$

The convex sum property of the weighting functions allows to write:

$$
\begin{array}{r}
\sum_{i, j, k=1}^{r} \mu_{i}(x) \mu_{j}(\hat{x}) \mu_{k}(x)\left(\tilde{x}^{T}\left(\mathscr{M}_{i j k}^{T} P+P \mathscr{M}_{i j k}\right) \tilde{x}\right. \\
\left.+\tilde{x}^{T} P \mathscr{B}_{i j k} u+u^{T} \mathscr{B}_{i j k}^{T} P \tilde{x}+\tilde{x}^{T} H^{T} H \tilde{x}-\gamma^{2} u^{T} u\right)<0
\end{array}
$$

which can be written in the matrix form:

$$
\sum_{i, j, k=1}^{r} \mu_{i}(x) \mu_{j}(\hat{x}) \mu_{k}(x) \tilde{r}^{T} \Xi_{i j k} \tilde{r}<0
$$

where:

$$
\begin{aligned}
\Xi_{i j k} & =\left[\begin{array}{cc}
\mathscr{M}_{i j k}^{T} P+P \mathscr{M}_{i j k}+H^{T} H & P \mathscr{B}_{i j k} \\
\mathscr{B}_{i j k}^{T} P & -\gamma^{2} I
\end{array}\right] \\
\tilde{r} & =\left[\begin{array}{c}
\tilde{x} \\
u
\end{array}\right]
\end{aligned}
$$

A sufficient condition for (29) to hold is:

$$
\begin{gathered}
{\left[\begin{array}{cc}
\mathscr{M}_{i j k}^{T} P+P \mathscr{M}_{i j k}+H^{T} H & P \mathscr{B}_{i j k} \\
\mathscr{B}_{i j k}^{T} P & -\gamma^{2} I
\end{array}\right]<0} \\
\forall(i, j, k) \in\{1, \ldots, r\}^{3}
\end{gathered}
$$

Let us choose the following structure for the matrix $P$ :

$$
P=\left[\begin{array}{cc}
P_{1} & 0 \\
0 & P_{2}
\end{array}\right]
$$

Using the definition of $\mathscr{M}_{i j k}$ and $\mathscr{B}_{i j k}$ given in (16), and the use of the changes of variables:

$$
K_{j}=P_{1} G_{j}
$$

and

$$
\bar{\gamma}=\gamma^{2}
$$

we obtain from (32) the LMI conditions expressed in (17) in the theorem 1 .

In many practical situations, the output is given by a set of sensors measuring a subset of the state variables. Assuming that the location of the sensors does not depend on the operating point, we have $D_{1}=D_{2}=\ldots=D_{r}=0$ and $C_{1}=C_{2}=\ldots=C_{r}=C$. The output of the system is then given by:

$$
y=C x
$$

In this case, the system (14) becomes:

$$
\begin{aligned}
\dot{\tilde{x}} & =\sum_{i, j=1}^{r} \mu_{i}(x) \mu_{j}(\hat{x})\left(\mathscr{M}_{i j} \tilde{x}+\mathscr{B}_{i j} u\right) \\
z & =H \tilde{x}
\end{aligned}
$$


where:

$$
\mathscr{M}_{i j}=\left[\begin{array}{cc}
A_{j}-G_{j} C & \Delta A_{i j}-G_{j} C \\
0 & A_{i}
\end{array}\right], \mathscr{B}_{i j}=\left[\begin{array}{c}
\Delta B_{i j} \\
B_{i}
\end{array}\right]
$$

The simplified version of theorem 1 is then given in the corollary 1.

Corollary 1: The system (37) is stable and the $\mathscr{L}_{2}$-gain of the transfer of $u(t)$ to $z(t)$ is bounded, if there exists symmetric matrices $P_{1}$ and $P_{2}$, matrices $K_{i}$ and a positive scalar $\bar{\gamma}$, such that the following conditions hold $\forall(i, j) \in$ $\{1, \ldots, r\}^{2}$ :

$$
\left[\begin{array}{ccc}
X_{1 j} & \Theta_{i j} & \Psi_{i j} \\
\Theta_{i j}^{T} & X_{2 i} & P_{2} B_{i} \\
\Psi_{i j}^{T} & B_{i}^{T} P_{2} & -\bar{\gamma} I
\end{array}\right]<0
$$

where:

$$
\begin{aligned}
X_{1 j} & =A_{j}^{T} P_{1}+P_{1} A_{j}-K_{j} C-C^{T} K_{j}^{T}+I \\
X_{2 i} & =A_{i}^{T} P_{2}+P_{2} A_{i} \\
\Theta_{i j} & =P_{1} \Delta A_{i j}-K_{j} C \\
\Psi_{i j} & =P_{1} \Delta B_{i j}
\end{aligned}
$$

The gains of the observer are derived from:

$$
G_{j}=P_{1}^{-1} K_{j}
$$

and the attenuation level is given by:

$$
\gamma=\sqrt{\bar{\gamma}}
$$

The performances of the observer can be increased by eigenvalue assignment in a specific region in the complex plane. For nonlinear Takagi-Sugeno systems, it is necessary to assign all of the eigenvalues of the local observers. The performance objectives tackled in the present paper are twofold: a decay rate to ensure the fast convergence of the observer and the limitation of the imaginary part of the eigenvalues of the observer to reduce the oscillatory phenomenon. Consequently, the eigenvalues of the system generating the state estimation error are clustered in an LMI region $S(\alpha, \beta)$ which is the intersection between a circle with center $(0,0)$ and radius $\beta$ and a strip with real part smaller than $-\alpha$. The corollary 2 gives the conditions of convergence of the observer taking into account the eigenvalues assignment.

Corollary 2: The system (37) is stable and the poles of system generating the state estimation error lie in $S(\alpha, \beta)$ and the $\mathscr{L}_{2}$-gain of the transfer from $u(t)$ to $z(t)$ is bounded, if there exist symmetric matrices $P_{1}$ and $P_{2}$, matrices $K_{i}$ and a positive scalar $\bar{\gamma}$, such that the following conditions hold:

$$
\begin{gathered}
{\left[\begin{array}{ccc}
X_{1 j} & \Theta_{i j} & \Psi_{i j} \\
\Theta_{i j}^{T} & X_{2 i} & P_{2} B_{i} \\
\Psi_{i j}^{T} & B_{i}^{T} P_{2} & -\bar{\gamma} I
\end{array}\right]<0, \in\{1, \ldots, r\}^{2}} \\
{\left[\begin{array}{cc}
-\beta P & A_{j}^{T} P-C^{T} K_{j}^{T} \\
P A_{j}-K_{j} C & -\beta P
\end{array}\right]<0} \\
A_{j}^{T} P+P A_{j}-C^{T} K_{j}^{T}-K_{j} C+2 \alpha P<0
\end{gathered}
$$

where $X_{1 j}, X_{2 i}, \Theta_{i j}$ and $\Psi_{i j}$ are defined in corollary 1. The gains of the observer are derived from:

$$
G_{j}=P_{1}^{-1} K_{j}
$$

and the attenuation level is given by:

$$
\gamma=\sqrt{\bar{\gamma}}
$$

Proof: The proof is similar to the proof of the theorem 1 using the results of eigenvalues assignment published in [16].

\section{STATE AND UNKNOWN INPUT ESTIMATION}

In this section, the problem of state and unknown input estimation is considered by extending the PI observer developed for linear systems to the case of TS systems with unmeasurable premise variables. Firstly time, the unknown input is assumed to be a constant signal. The convergence conditions are obtained by using the results given in the previous sections. Secondly, the derivative of the unknown input is assumed to be bounded. The convergence conditions are given in the LMIs formulation. For the sake of simplicity, the output of the considered systems is linear with respect to the state, the input and the unknown input $\left(C_{1}=\ldots=C_{r}=C\right.$, $D_{1}=\ldots=D_{r}=D$ and $\left.F_{1}=\ldots=F_{r}=F\right)$.

Let us consider the following system:

$$
\left\{\begin{array}{l}
\dot{x}=\sum_{i=1}^{r} \mu_{i}(x)\left(A_{i} x+B_{i} u+E_{i} f\right) \\
y=C x+D u+F f
\end{array}\right.
$$

Assumption 1: The following assumption holds :

$$
\dot{f}(t)=0
$$

Let us consider the augmented state $x_{a}=\left[\begin{array}{ll}x^{T} & f^{T}\end{array}\right]^{T}$, then the corresponding augmented system is:

$$
\left\{\begin{array}{l}
\dot{x}_{a}=\sum_{i=1}^{r} \mu_{i}(x)\left(\tilde{A}_{i} x_{a}+\tilde{B}_{i} u\right) \\
y=\tilde{C} x_{a}+D u
\end{array}\right.
$$

where:

$$
\tilde{A}_{i}=\left[\begin{array}{cc}
A_{i} & E_{i} \\
0 & 0
\end{array}\right], \tilde{B}_{i}=\left[\begin{array}{c}
B_{i} \\
0
\end{array}\right], \tilde{C}=\left[\begin{array}{ll}
C & F
\end{array}\right]
$$

Applying the same method used to obtain (7)-(8), the system (54) can be transformed into the following equivalent form:

$$
\dot{x}_{a}=\sum_{i, j=1}^{r} \mu_{i}(x) \mu_{j}(\hat{x})\left(\left(\tilde{A}_{j}+\Delta \tilde{A}_{i j}\right) x_{a}+\left(\tilde{B}_{j}+\Delta \tilde{B}_{i j}\right) u\right)
$$

where:

$$
\Delta X_{i j}=X_{i}-X_{j}, \quad X_{i} \in\left\{\tilde{A}_{i}, \tilde{B}_{i}\right\}
$$

The PI observer is given by:

$$
\left\{\begin{array}{l}
\dot{\hat{x}}_{a}=\sum_{j=1}^{r} \mu_{j}(\hat{x})\left(\tilde{A}_{j} \hat{x}_{a}+\tilde{B}_{j} u+\tilde{G}_{j}(y-\hat{y})\right) \\
\hat{y}=\tilde{C} \hat{x}_{a}+D u
\end{array}\right.
$$

where:

$$
\tilde{G}_{j}=\left[\begin{array}{c}
G_{P j} \\
G_{I j}
\end{array}\right]
$$


The estimation error is given by $e_{a}=x_{a}-\hat{x}_{a}$, and its dynamics is given by:

$$
\begin{aligned}
\dot{e}_{a} & =\sum_{i, j=1}^{r} \mu_{i}(x) \mu_{j}(\hat{x})(\underbrace{\left(\tilde{A}_{j}-\tilde{G}_{j} \tilde{C}\right)}_{\Phi_{j}} e_{a}+\Delta \tilde{A}_{i j} x_{a}+\Delta \tilde{B}_{i j} u) \\
& =\sum_{i, j=1}^{r} \mu_{i}(x) \mu_{j}(\hat{x})\left(\Phi_{j} e_{a}+\left[\begin{array}{c}
\Delta A_{i j} \\
0
\end{array}\right] x\right. \\
& \left.+\left[\begin{array}{c}
\Delta E_{i j} \\
0
\end{array}\right] f+\Delta \tilde{B}_{i j} u\right) \\
& =\sum_{i, j=1}^{r} \mu_{i}(x) \mu_{j}(\hat{x})\left(\Phi_{j} e_{a}+\left[\begin{array}{c}
\Delta A_{i j} \\
0
\end{array}\right] x+\tilde{\Gamma}_{i j} \omega\right)
\end{aligned}
$$

where:

$$
\tilde{\Gamma}_{i j}=\left[\begin{array}{cc}
\Delta \tilde{E}_{i j} & \Delta \tilde{B}_{i j}
\end{array}\right], \quad \omega=\left[\begin{array}{l}
f \\
u
\end{array}\right], \quad \Delta \tilde{E}_{i j}=\left[\begin{array}{c}
\Delta E_{i j} \\
0
\end{array}\right]
$$

Let us define the augmented state $\tilde{x}=\left[\begin{array}{ll}e_{a}^{T} & x^{T}\end{array}\right]^{T}$, then the augmented system is:

$$
\begin{gathered}
\dot{\tilde{x}}=\sum_{i, j=1}^{r} \mu_{i}(x) \mu_{j}(\hat{x})(\underbrace{\left[\begin{array}{cc}
\Phi_{j} & \Delta A_{i j} \\
0 & A_{i}
\end{array}\right]}_{M_{i j}} \tilde{x}+\underbrace{\left[\begin{array}{c}
\tilde{\Gamma}_{i j} \\
\bar{B}_{i}
\end{array}\right]}_{R_{i j}} \omega) \\
z=H \tilde{x}
\end{gathered}
$$

where:

$$
\bar{B}_{i}=\left[\begin{array}{ll}
E_{i} & B_{i}
\end{array}\right]
$$

The system (60) has the same form than (37). The objective is to assure the stability of (60) while minimizing the influence of $\omega(t)$ on the estimation error $z(t)$. By using the corollary 1 , we obtain the sufficient convergence conditions of the PI observer (56).

Theorem 2: The system (60) is stable and the $\mathscr{L}_{2}$-gain of the transfer from $\omega(t)$ to $z(t)$ is bounded, if there exists symmetric matrices $P_{1}$ and $P_{2}$, matrices $\tilde{G}_{i}$ and a positive scalar $\bar{\gamma}$, such that the following conditions hold $\forall(i, j) \in$ $\{1, \ldots, r\}^{2}$ :

$$
\left[\begin{array}{cccc}
X_{1 j} & \Theta_{i j} & P_{1} \Delta \tilde{E}_{i j} & P_{1} \Delta \tilde{B}_{i j} \\
\Theta_{i j}^{T} & X_{2 i} & P_{2} E_{i} & P_{2} B_{i} \\
\Delta \tilde{E}_{i j}^{T} P_{1} & E_{i}^{T} P_{2} & -\bar{\gamma} I & 0 \\
\Delta \tilde{B}_{i j}^{T} P_{1} & B_{i}^{T} P_{2} & 0 & -\bar{\gamma} I
\end{array}\right]<0
$$

where:

$$
\begin{aligned}
X_{1 j} & =\tilde{A}_{j}^{T} P_{1}+P_{1} \tilde{A}_{j}-L_{j} \tilde{C}-\tilde{C}^{T} L_{j}^{T}+I \\
X_{2 i} & =A_{i}^{T} P_{2}+P_{2} A_{i} \\
\Theta_{i j} & =P_{1} \Delta \tilde{A}_{i j}
\end{aligned}
$$

The gains of the observer are derived from:

$$
\tilde{G}_{j}=\left[\begin{array}{c}
G_{P j} \\
G_{I j}
\end{array}\right]=P_{1}^{-1} L_{j}
$$

and the attenuation level is given by:

$$
\gamma=\sqrt{\bar{\gamma}}
$$

The Proportional Integral Observer (PIO) can be applied only in the case where the unknown input is constant or slowly time varying. In order to estimate other types of unknown inputs, the Proportional Multiple Integral Observer (PMIO) is proposed in [17].

\section{Simulation RESUlts}

To illustrate the effectiveness of the proposed methods, numerical examples are given in this section. The first one concerns the state estimation and the second one uses the PI observer in order to estimate the state and the unknown inputs.

\section{A. State estimation}

Consider the system described in (1) defined by:

$$
\begin{gathered}
A_{1}=\left[\begin{array}{ccc}
-2 & 1 & 1 \\
1 & -3 & 0 \\
2 & 1 & -2
\end{array}\right], A_{2}=\left[\begin{array}{ccc}
-3 & 2 & -2 \\
5 & -3 & 0 \\
1 & 2 & -4
\end{array}\right] \\
B_{1}=\left[\begin{array}{c}
1 \\
0.5 \\
0.25
\end{array}\right], B_{2}=\left[\begin{array}{c}
1.5 \\
3 \\
2
\end{array}\right], C=\left[\begin{array}{lll}
1 & 1 & 1 \\
1 & 0 & 1
\end{array}\right]
\end{gathered}
$$

The weighting functions are defined by:

$$
\left\{\begin{array}{l}
\mu_{1}(x)=\frac{1-\tanh \left(x_{1}\right)}{2} \\
\mu_{2}(x)=1-\mu_{1}(x)=\frac{1+\tanh \left(x_{1}\right)}{2}
\end{array}\right.
$$

By using the corollary 2 with eigenvalues assignment in an LMI region defined by $\alpha=0.1$ and $\beta=10$, we obtain the following matrices:

$$
L_{1}=\left[\begin{array}{cc}
-1.556 & 8.556 \\
6.919 & -8.956 \\
-1.684 & 6.472
\end{array}\right], L_{2}=\left[\begin{array}{cc}
-1.556 & 8.556 \\
6.919 & -8.956 \\
-1.684 & 6.472
\end{array}\right]
$$

An output noise bounded by 1 is added to the output of the system in order to simulate measurement noise. The simulation results in the figure 1 illustrate the convergence of the state estimation error in the UI free case.
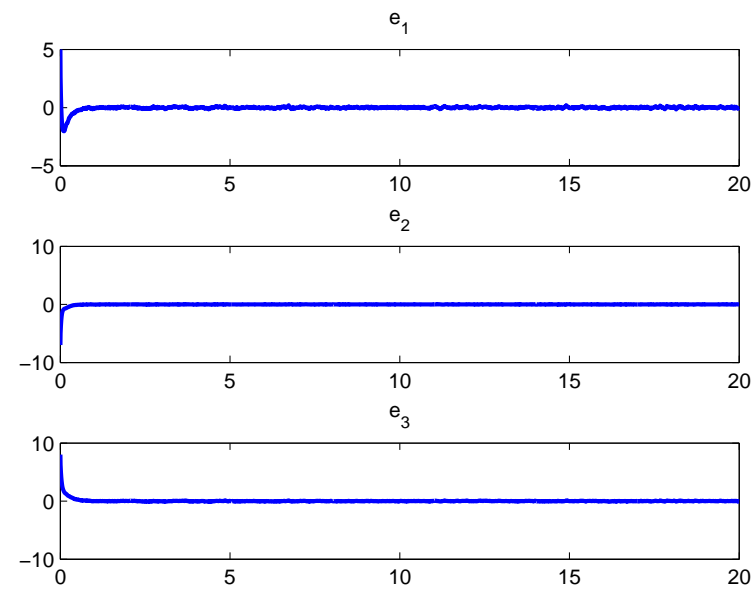

Fig. 1. State estimation errors 


\section{B. State and unknown input estimation}

In this section, actuator and sensor faults are added to the previous example. Their respective influences are defined by the following matrices:

$$
E_{1}=\left[\begin{array}{ll}
1 & 0 \\
0 & 0 \\
1 & 0
\end{array}\right], E_{2}=\left[\begin{array}{ll}
1 & 0 \\
1 & 0 \\
1 & 0
\end{array}\right], G=\left[\begin{array}{ll}
0 & 1 \\
0 & 1
\end{array}\right]
$$

The simulation results of the UI estimation obtained by the PI observer are displayed on figure 2. Solving the LMIs in the theorem 2 may cause slow dynamics of the observer, so an eigenvalue assignment in the same region as the previous simulation allows to increase the performances of the observer.

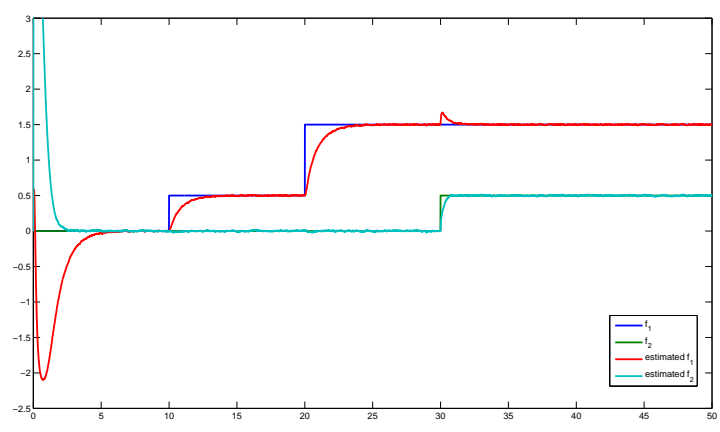

Fig. 2. Unknown inputs

\section{CONCLUSION}

This paper addresses new methods to design observers for nonlinear systems described by Takagi-Sugeno models with unmeasurable premise variables. The first observer is devoted to the estimation of the state of a given system and the second observer which is an extension of the classical Proportional Integral Observer (PIO) developed for linear systems, provides both the estimation of the state variables and of the UI. The proposed approach is based on some transformations using the convex property of the sum of the weighting functions. The convergence of the estimation errors are studied using the second method of Lyapunov and the $\mathscr{L}_{2}$ techniques, and the conditions which guarantee the convergence of the estimation errors are obtained in the Linear Matrix Inequalities (LMIs) formulation. The future works will concern, on the one hand, the application of the proposed methods to develop a scheme for diagnosis of nonlinear systems, and on the other hand, the convergence conditions will be studied in order to reduce its conservatism, by using for example, other kinds of Lyapunov functions.

\section{REFERENCES}

[1] T. Takagi and M. Sugeno, "Fuzzy identification of systems and its applications to modeling and control," IEEE Transactions on Systems, Man, and Cybernetics, vol. 15, pp. 116-132, 1985.

[2] K. Tanaka, T. Ikeda, and H. Wang, "Fuzzy regulators and fuzzy observers: Relaxed stability conditions and LMI-based designs," IEEE Transactions on Fuzzy Systems, vol. 6, no. 2, pp. 250-265, 1998.
[3] M. Chadli, D. Maquin, and J. Ragot, "Non quadratic stability analysis of Takagi-Sugeno systems," in IEEE Conference on Decision and Control, CDC'2002, Las Vegas, Nevada, USA, 2002.

[4] A. Akhenak, M. Chadli, J. Ragot, and D. Maquin, "Design of sliding mode unknown input observer for uncertain Takagi-Sugeno model," in 15th Mediterranean Conference on Control and Automation, MED'07, Athens, Greece, 2007.

[5] M. Rodrigues, D. Theilliol, M. Adam-Medina, and D. Sauter, "A fault detection and isolation scheme for industrial systems based on multiple operating models," Control Engineering Practice, vol. 16, no. 2, pp. 225-239, Feb. 2008.

[6] M. El-Ghatwary, S. Ding, and Z. Gao, "Robust fuzzy fault detection for continuous-time nonlinear dynamic systems," in IFAC Symp. SAFEPROCESS, Beijing, China, 2006.

[7] P. Bergsten, R. Palm, and D. Driankov, "Observers for Takagi-Sugeno fuzzy systems," IEEE Transactions on Systems, Man, and Cybernetics - Part B: Cybernetics, vol. 32, no. 1, pp. 114-121, 2002.

[8] — "Fuzzy observers," in IEEE International Fuzzy Systems Conference, Melbourne Australia, 2001.

[9] P. Bergsten and R. Palm, "Thau-Luenberger observers for TS fuzzy systems," in 9th IEEE International Conference on Fuzzy Systems, FUZZ IEEE 2000, San Antonio, TX, USA, 2000.

[10] F. Thau, "Observing the state of non-linear dynamic systems," International Journal of Control, vol. 18, pp. 471-479, 1973.

[11] K. Tanaka and H. Wang, Fuzzy Control Systems Design and Analysis: A Linear Matrix Inequality Approach, J. Wiley and i. Sons, Eds. John Wiley and Sons, inc, 2001.

[12] T. Guerra, A. Kruszewski, L. Vermeiren, and H. Tirmant, "Conditions of output stabilization for nonlinear models in the Takagi-Sugeno's form," Fuzzy Sets and Systems, vol. 157, no. 9, pp. 1248-1259, May 2006 .

[13] A. Kruzewski, R. Wang, and T. Guerra, "Non-quadratic stabilization conditions for a class of uncertain non linear discrete-time T-S fuzzy models: a new approach," IEEE Transactions on Automatic Control, vol. 53, no. 2, pp. 606-611, 2008.

[14] R. Patton, J. Chen, and C. Lopez-Toribio, "Fuzzy observers for nonlinear dynamic systems fault diagnosis," in 37th IEEE Conference on Decision and Control, Tampa, Florida USA, 1998.

[15] R. Palm and P. Bergsten, "Sliding mode observers for Takagi-Sugeno fuzzy systems." 9th IEEE International Conference on Fuzzy Systems, FUZZ IEEE 2000, San Antonio, TX, USA, 2000.

[16] M. Chilali and P. Gahinet, "H-infinity design with pole placement constraints : an LMI approach," IEEE Transactions on Automatic Control, vol. 41, no. 3, pp. 358-367, 1996.

[17] D. Ichalal, B. Marx, J. Ragot, and D. Maquin, "Simultaneous state and unknown inputs estimation with pi and pmi observers for takagisugeno model with unmeasurable premise variables." in 17th Mediterranean Conference on Control and Automation, MED'09, Thessaloniki, Greece, June 24-26 2009. 